\title{
A regionalização no contexto atual das políticas de saúde
}

\author{
Regionalization in the current context \\ of health policies
}

Renilson Rehem de Souza 1

\footnotetext{
1 Secretaria de Assistência à Saúde do Ministério da Saúde. Esplanada dos Ministérios,

bloco G, 9o andar 70058-900, Brasília DF. renilson@saude.gov.br
}

\begin{abstract}
This article deals with the management of the decentralization process in the Brazilian Unified Health System (SUS) in the 1990s, relating it to the establishment and application of the so-called Basic Operational Norms (NOB) in force from 1991 to 1996. The author highlights the usefulness of these instruments for orienting the three levels of government and their negotiation, while also discussing their limits, particularly those pertaining to the complexity and different degrees of progress experienced by the various States and municipalities in implementing the Unified Health System. For this very reason he contends that the NOBs have run their course in terms of the current dynamics of health sector decentralization. He focuses attention on another instrument, the so-called Health Care Operational Norm (NOAS SUS 01/01), around which a working consensus is currently being sought among the key actors in health sector reform: the Ministry of Health, the Bipartisan and Tripartisan Committees, and the National Health Council. Based on the critique of the NOB-96, the author favors further promoting the logic of guaranteeing the population's access to health services and programs at all levels.

Key words Decentralization, Implementation of the Unified Health System, Public sector reform
\end{abstract}

Resumo Este artigo trata da gestão do processo de descentralização do SUS na década de 1990, relacionando-o à construção e à aplicação das Normas Operacionais Básicas (NOB) que vigoraram de 1991 a 1996. O autor valoriza esses instrumentos de orientação para as três esferas de governo e sua "pactuação", mas também discute os seus limites, sobretudo, relacionados à complexidade e às diferenciações de avanço dos estados e dos municípios, no processo de implantação do SUS. Por isso mesmo considera o esgotamento das NOBs para a situação presente da dinâmica da descentralização setorial. Sua atenção se centra em outro instrumento: Norma Operacional de Assistência à Saúde (NOAS SUS 01/01) para a qual se busca um consenso mínimo entre todos os atores responsáveis pela reforma setorial: o Ministério, as Comissões Bipartites e Tripartites e o Conselho Nacional de Saúde. A partir da crítica da NOB-96, o autor propõe que se acentue a lógica de garantia de acesso da população às ações e aos serviços de saúde em todos os níveis de assistência.

Palavras-chave Descentralização, Implementação do SUS, Reforma do Estado 


\section{O processo de descentralização da gestão do SUS na década de 1990}

Um dos principais avanços da implementação do SUS, ao longo da década de 1990, se relaciona ao acelerado processo de descentralização político-administrativa, com progressiva transferência de responsabilidades e recursos do nível federal para os gestores estaduais e municipais.

As Normas Operacionais do SUS têm representado um importante instrumento de regulamentação desse processo de descentralização, à medida que estabelecem, de forma negociada, mecanismos e critérios para a transferência de responsabilidades e recursos para estados e municípios.

Ao longo da década de 1990, foram editadas quatro dessas normas - as NOB 01/91, NOB 01/92, NOB 01/93 e NOB 01/96, sendo que as duas últimas foram resultantes de processos de negociação progressivamente mais intensos entre os atores setoriais, particularmente no âmbito da Comissão Intergestores Tripartite e do Conselho Nacional de Saúde.

A descentralização impulsionada por essas normas apresentou resultados positivos relacionados, entre outros fatores, ao expressivo aumento de transferências diretas de recursos do nível federal para o estadual e municipal, ${ }^{1}$ ao fortalecimento da capacidade institucional de diversos estados e municípios na gestão da saúde e à expansão da rede de serviços municipais de saúde.

Por outro lado, o próprio avanço da descentralização evidenciou a necessidade de enfrentamento de um conjunto de problemas, tanto no âmbito da gestão, quanto no que se refere à atenção à saúde.

Em relação à gestão, diversos estados ainda não assumiram plenamente as funções de coordenação e de regulação dos sistemas municipais de saúde e das redes intermunicipais de atenção. Além disso, o pequeno porte da maior parte dos municípios brasileiros traz dificuldades no sentido da capacidade de planejamento do sistema, da regulação de prestadores e da construção de redes assistenciais adequadas para o atendimento da população, já que não é possível garantir a oferta de serviços de média e alta complexidade em todos os municípios brasileiros.

O dispositivo - incluído nas disposições transitórias da NOB 96 no processo de negociação da proposta para viabilizar sua aprova- ção -, que delega às CIBs a competência para definir partilhas de gestão diferentes do propugnado comando único sobre os prestadores, possibilitou o estabelecimento de pactos de gestão entre estados e municípios pouco adequados, gerando situações de competição predatória, em detrimento da integração dos papéis desempenhados por cada gestor, fragilizando o comando efetivamente público sobre o sistema e a subordinação dos prestadores às necessidades de saúde identificadas pelos gestores.

O financiamento do sistema, apesar dos avanços obtidos, ainda permanece, em muitos casos, fortemente vinculado à lógica da oferta de serviços, atrelada à série histórica de gastos e de produção e à capacidade instalada existente, o que mantém a concentração de recursos nas áreas mais desenvolvidas e em ações nem sempre compatíveis com o perfil de necessidades da população. No âmbito dos estados, os critérios adotados para a distribuição de recursos entre os municípios, em geral, são pouco explícitos. Muitos estados sequer estabeleceram os tetos financeiros para todos os seus municípios, deixando essa definição para o momento de habilitação em Gestão Plena do Sistema Municipal - GPSM, quando se torna potencialmente geradora de conflitos. O comando da utilização dos recursos determinado pelo mercado existente de serviços dificulta a seleção de prioridades de intervenção e a reorganização da rede de unidades, produzindo a manutenção das iniqüidades distributivas e da ineficiência alocativa.

Outro ponto que demanda melhor equacionamento refere-se ao planejamento e organização funcional do sistema: embora muitos estados tenham formalmente conduzido processos de "Programação Pactuada e Integrada" - PPI, sua implementação tem sido efetiva em poucos casos, dificultando a integração intermunicipal. As limitações da capacidade de planejamento e coordenação das SES aumentam o risco de atomização dos sistemas municipais e de conseqüências indesejáveis, como a incorporação tecnológica irracional e implantação de barreiras de acesso a residentes em outros municípios.

No que se refere ao modelo assistencial, o aumento da resolutividade e a garantia de acesso aos serviços em todos os níveis de complexidade se colocam como desafios: a configuração do elenco de procedimentos incluídos na atenção básica (Piso de Atenção Básica fixo 
- PAB fixo) mostrou-se restrita, apresentando baixa capacidade de resolução dos problemas mais freqüentes dos usuários. Simultaneamente, ocorrem dificuldades de acesso à assistência de média e de alta complexidade, em especial face à enorme concentração desses serviços em poucos municípios e, mesmo a completa inexistência de determinados serviços de alta complexidade em diversos estados. O instrumental operacional utilizado para referências intermunicipais e interestaduais tem sido insuficiente para garantir o acesso.

Além dos limites no âmbito da gestão e da reorientação do modelo assistencial, o processo de habilitação das SMS e de aumento das transferências automáticas "fundo a fundo" começou a demonstrar sinais de esgotamento, sendo residual o número de municípios que ainda não se encontra em uma das condições de gestão previstas na NOB 96. A taxa de crescimento do volume de recursos transferidos "fundo a fundo", bastante significativa no primeiro ano de vigência da referida norma, apresentou uma desaceleração no período de dezembro de 1998 a dezembro de 2000, indicando a importância de se desenvolver novos mecanismos para ampliar a descentralização dos recursos financeiros e buscar a superação progressiva dos mecanismos de repasse entre gestores por produção de serviços.

\section{A Norma Operacional de Assistência à Saúde (NOAS SUS 01/01): descentralização com ênfase na regionalização}

No contexto de análise dos avanços e limitações da descentralização nos anos 90, a questão da regionalização passa a se destacar no debate setorial ao final da década, particularmente no âmbito da Comissão Intergestores Tripartite (CIT) e do Conselho Nacional de Saúde (CNS). Os grandes avanços no âmbito da descentralização político-administrativa, com fortalecimento dos gestores locais e as mudanças na organização da atenção básica induzidas pela NOB SUS 01/96, evidenciam a necessidade de maior articulação entre os sistemas municipais e de fortalecimento das secretarias estaduais de saúde na sua função reguladora para assegurar a organização de redes assistenciais regionalizadas, hierarquizadas e resolutivas, que propiciem resultados positivos para a saúde da população.
A publicação da Norma Operacional de Assistência à Saúde - NOAS SUS 01/01, em janeiro de 2001, foi fruto de cerca de um ano de debates entre o Ministério da Saúde, as representações nacionais dos secretários estaduais e municipais de saúde e o Conselho Nacional de Saúde. Dessa forma, não é correto afirmar que a NOAS-SUS 01/01 seja uma normatização definida unilateralmente pelo Ministério da Saúde. Pelo contrário, ela traduz o resultado de um amplo e franco processo de construção de um "consenso mínimo" sobre os principais desafios e as correspondentes estratégias de enfrentamento a serem adotadas pelos gestores. Esse caráter de construção conjunta da NOASSUS $01 / 01$ pode ser constatado pela análise das sucessivas versões do documento produzidas no período.

O objetivo geral da NOAS SUS 01/01 é: "Promover maior eqüidade na alocação de recursos e no acesso da população às ações e serviços de saúde em todos os níveis de atenção" (MS, 2001).

Para atingir esse objetivo, a NOAS define a regionalização como macroestratégia de reorganização assistencial: “A macroestratégia de regionalização deverá contemplar uma lógica de planejamento integrado de maneira a conformar sistemas funcionais de saúde, ou seja, redes articuladas e cooperativas de atenção, referidas a territórios delimitados e a populações definidas, dotadas de mecanismos de comunicação e fluxos de inter-relacionamento que garantam o acesso dos usuários às ações e serviços de níveis de complexidade necessários para a resolução de seus problemas de saúde, otimizando os recursos disponíveis" (MS, 2001).

A NOAS-SUS 01/01 aborda três grupos de estratégias articuladas que visam contribuir para o processo de regionalização em saúde, como forma de promover a descentralização com eqüidade no acesso.

- Elaboração do Plano Diretor de Regionalização e diretrizes para a organização regionalizada da assistência, visando à conformação de sistemas de atenção funcionais e resolutivos nos diversos níveis.

- Fortalecimento das capacidades gestoras do SUS, que compreende um conjunto de estratégias voltadas para consolidar o caráter público da gestão do sistema, por meio da instrumentalização dos gestores estaduais e municipais para o desenvolvimento de funções como planejamento/programação, regulação, con- 
trole e avaliação, incluindo instrumentos de consolidação de compromissos entre gestores. - Atualização dos critérios e do processo de habilitação de estados e municípios às condições de gestão do SUS, visando torná-lo coerente com o conjunto de mudanças propostas.

Um dos pontos mais importantes da NOAS SUS 01/01 diz respeito ao processo de elaboração do Plano Diretor de Regionalização, coordenado pelo gestor estadual, com a participação do conjunto de municípios. Esse Plano, a ser aprovado pelo Conselho Estadual de Saúde, deve conter minimamente: (a) a divisão do território estadual em regiões/microrregiões de saúde, definidas segundo critérios sanitários, epidemiológicos, geográficos, sociais, de oferta de serviços e de acessibilidade; (b) o diagnóstico dos principais problemas de saúde e das prioridades de intervenção; (c) a constituição de módulos assistenciais resolutivos, formados por um ou mais municípios, que dêem conta do primeiro nível da média complexidade, visando garantir o suporte às ações de Atenção Básica; (d) os fluxos de referência para todos os níveis de complexidade e os mecanismos de relacionamento intermunicipal; (e) o Plano Diretor de Investimentos, que procura suprir as lacunas assistenciais identificadas, de acordo com as prioridades de intervenção.

No que diz respeito à organização da assistência, enfatiza-se a importância de qualificar e melhorar a resolutividade da atenção básica em todos os municípios brasileiros, a partir da identificação de áreas estratégicas mínimas, relacionadas a problemas de saúde de abrangência nacional (saúde da mulher, saúde da criança, saúde bucal, controle da hipertensão e diabetes, controle da tuberculose e eliminação da hanseníase). Complementarmente, os gestores estaduais e municipais podem definir outras áreas estratégicas, de acordo com as especificidades epidemiológicas locais.

Além das ações mínimas de atenção básica a serem asseguradas em todos os municípios brasileiros, independente de seu porte populacional, a NOAS SUS $01 / 01$ propõe a formação de módulos assistenciais resolutivos, constituídos por um ou mais municípios, que garantam no âmbito microrregional o acesso ágil e oportuno de todos os cidadãos a um conjunto de ações de saúde freqüentemente necessárias para atender os problemas de saúde mais comuns, que nem sempre podem ser oferecidas em todos os municípios pelo seu peque- no porte populacional. ${ }^{2}$ A proposta de qualificação de regiões/microrregiões na assistência à saúde apresentada na referida Norma se fundamenta, portanto, na busca de garantia de acesso a ações resolutivas para além dos limites municipais, considerando critérios de qualidade e economia de escala.

Ainda no que tange à assistência à saúde, a NOAS SUS 01/01 estabelece diretrizes gerais para a organização das demais ações de média e alta complexidade, e preconiza que o plano de regionalização compreenda o mapeamento das redes de referência em áreas estratégias específicas (gestação de alto risco, urgência e emergência, hemoterapia, entre outras).

Esse tipo de processo de regionalização, incentivado pela NOAS SUS 01/01, requer a articulação dos gestores municipais para a negociação e pactuação de referências intermunicipais, sob coordenação e regulação estadual, que deve se dar através da programação pactuada e integrada. O Ministério da Saúde, por sua vez, deverá estabelecer mecanismos de garantia de acesso aos serviços de alta complexidade que não estiverem disponíveis em todas as unidades da federação. Além disso, faz-se necessário o fortalecimento da capacidade gestora dos três níveis de governo para exercer as funções de regulação, controle e avaliação do sistema, em uma nova perspectiva.

Do ponto de vista do financiamento, a NOAS SUS 01/01 propõe um aumento do componente de financiamento federal calculado em uma base per capita, ao propor uma ampliação do Piso de Atenção Básica - fixo e que o financiamento das ações do primeiro nível da média complexidade ambulatorial se dê com base em um valor per capita nacional. Esse aspecto é importante porque assinala uma tendência de superação da lógica anterior de financiamento, fortemente orientada pela oferta preexistente de serviços, a partir da expansão dos mecanismos de pré-pagamento, que requerem um papel mais ativo dos gestores no planejamento da oferta, de acordo com as necessidades da população e prioridades identificadas.

\section{Perspectivas de implementação da NOAS-SUS 01/2001}

Os diversos estados brasileiros se encontram em momentos muito diferentes no que diz respeito ao processo de descentralização e regionalização. Enquanto em alguns estados per- 
sistem ainda modelos com baixa descentralização de ações e serviços para os municípios; em outros, a municipalização se concretizou efetivamente e há avanços expressivos no sentido da articulação entre os municípios e organização de redes regionalizadas e hierarquizadas.

O Ministério da Saúde vem acompanhando e apoiando a regionalização nos estados nesse momento de implementação da NOAS SUS 01/01, considerando as especificidades de cada um. Além do apoio técnico à elaboração dos planos de regionalização e da programação integrada, está previsto um aporte adicional de recursos federais para o custeio de expansão da oferta e para a implementação de um valor per capita nacional para o primeiro nível da média complexidade.

Espera-se que, até o final de 2001, os estados encaminhem à Comissão Intergestores Tripartite seus Planos Diretores de Regionalização, aprovados pelos respectivos conselhos de saúde, e os produtos da PPI.

$\mathrm{O}$ alcance pleno do objetivo fundamental da estratégia de regionalização desencadeada pela NOAS - a garantia de acesso da população a todos os níveis de atenção necessários - certamente se coloca como um desafio a todos os atores setoriais que participaram do processo de elaboração da NOAS e que continuam participando ativamente de sua implantação. Afinal, os esforços de regulamentação obviamente apresentam limites. Por mais discutidas e negociadas que sejam em sua concepção, as normas por si mesmas não resolvem todos os conflitos de interesses legítimos dos atores relacionados ao processo de gestão do SUS. Além disso, a implementação das políticas de saúde, assim como das demais políticas públicas, é freqüentemente tensionada pela existência de interesses particularistas que podem colidir com o interesse público.

Nesse sentido, é importante avançarmos na operacionalização do consenso mínimo obtido no processo de elaboração da NOAS-SUS $01 / 01$, para que possamos dar mais um salto de qualidade em direção ao sistema de saúde que desejamos oferecer à sociedade brasileira. Os instrumentos disponibilizados pela NOAS-SUS 01/01 poderão contribuir decisivamente para a organização funcional do sistema de saúde e o fortalecimento da gestão pública no SUS, à medida que os atores do setor saúde comprometidos com a Reforma Sanitária brasileira se apropriem e avancem para além das diretrizes apresentadas

Em síntese, a NOAS SUS 01/01 é marcada pelos seguintes pontos, que procuram consolidar os princípios do SUS: (a) a necessidade de deslocar a ênfase do processo de descentralização para a garantia de acesso a ações e serviços de saúde em todos os níveis; (b) a importância de reafirmar o comando único em cada nível de governo, assegurando que o gestor municipal assuma a gestão do conjunto de ações e serviços em seu território, sempre que estiver plenamente preparado para tanto; (c) a premência de qualificar e reorientar o papel do gestor estadual, deslocando cada vez mais essa esfera das funções de prestação direta de serviços e de relação com prestadores, para as funções de formulação de políticas e planejamento/programação estadual, coordenação e regulação intermunicipal, controle e avaliação sistêmicas - incluindo análises de resultados e impacto das ações de saúde -, e capacitação e apoio técnico aos municípios.

\section{Notas}

\footnotetext{
1 Em dezembro de 2000, cerca de 99\% dos municípios brasileiros se encontravam habilitados a receber diretamente recursos federais para atenção à saúde, segundo as regras da Norma Operacional Básica do SUS 01/96. Dessa forma, entre os recursos federais da assistência, cerca de $63 \%$ já eram repassados diretamente para os fundos municipais ou fundos estaduais de saúde nesta data.

2 No ano de 1999 , cerca de 50\% dos municípios brasileiros apresentavam menos de 10.000 habitantes e mais de $80 \%$ dos municípios do país apresentavam menos de 30.000 habitantes. Vale assinalar que, em alguns estados, a proporção de pequenos municípios é bem superior à média nacional.
} 